\title{
Monte Carlo simulation of magnetization switching in a Heisenberg model for small ferromagnetic particles
}

\author{
D. Hinzke ${ }^{1}$, U. Nowak ${ }^{2}$ \\ Theoretische Tieftemperaturphysik, Gerhard-Mercator-Universität-Duisburg, 47048 Duisburg, Germany
}

\begin{abstract}
Using Monte Carlo methods we investigate the thermally activated magnetization switching of small ferromagnetic particles driven by an external magnetic field. For low uniaxial anisotropy one expects that the spins rotate coherently while for sufficiently large anisotropy the reversal should be due to nucleation. The latter case has been investigated extensively by Monte Carlo simulation of corresponding Ising models. In order to study the crossover from coherent rotation to nucleation we use a specially adjusted update algorithm for the Monte Carlo simulation of a classical three-dimensional Heisenberg model with a finite uniaxial anisotropy. This special algorithm which uses a combined sampling can simulate different reversal mechanisms efficiently. It will be described in detail and its efficiency and physical validity will be discussed by a comparison with other common update-algorithms.
\end{abstract}

Ferromagnetic particles are important for information storage in magnetic recording media (see, e.g., [1] for a review). Hence, much effort has been devoted to an understanding of the behavior of small magnetic particles experimentally [2-4], analytically [5], and in computer simulations. Thermally activated magnetization reversal by nucleation and subsequent domain wall motion has been studied mainly by Monte Carlo simulations of Ising models [6-10]. For other reversal mechanisms like coherent rotation it is necessary to study models with continuous degrees of freedom $[8,10,11]$. In these models an important issue for the efficiency of the Monte Carlo algorithm is the choice of the trial step of the single magnetic moments. In the following we will discuss different update-algorithms which can simulate thermally activated magnetization reversal in detail.

\footnotetext{
${ }^{1}$ E-mail: denise@thp.uni-duisburg.de.

${ }^{2}$ E-mail: uli@thp.uni-duisburg.de.
}

We will consider a classical three-dimensional Heisenberg model which is defined by the Hamiltonian

$\mathcal{H}=-J \sum_{\langle i j\rangle} \mathbf{S}_{i} \cdot \mathbf{S}_{j}-d \sum_{i}\left(S_{i}^{z}\right)^{2}-\mathbf{B} \cdot \sum_{i} \mathbf{S}_{i}$,

where the $\mathbf{S}_{i}$ are three-dimensional vectors of unit length. The first sum which represents the exchange of the spins is over nearest neighbor interactions with the ferromagnetic exchange coupling constant $J>$ 0 . The second sum represents a uniaxial anisotropy which favors the $z$-axis as easy axis (anisotropy constant $d>0$ ). The last sum is the coupling of the spins to an applied magnetic field, where $\mathbf{B}$ is the strength of the field times the absolute value of the magnetic moment of the spin. Using this model it is possible to investigate different reversal mechanisms of the system, coherent rotation as well as nucleation depending on the strength of the anisotropy constant.

We will investigate the thermally activated reversal of the magnetization of a particle. The particle is 
destabilized by an applied magnetic field which is pointing in the direction of the easy axis, antiparallel to the initial magnetization of the system. Due to the field the initial state is metastable so that after some time the particle will reverse its magnetization. During the reversal the particle has to overcome an energy barrier $\Delta E$ which is called the activation energy by thermal fluctuations. For sufficiently low temperatures the corresponding lifetime of the metastable state is then $[13,14]$

$\tau=\tau_{0} \exp \left(\frac{\Delta E}{T}\right)$.

The energy barrier $\Delta E$ depends on the details of the reversal mechanism. For high anisotropy it is energetically favorable for the system to form domain walls, i.e. the system divides into parts with opposite directions of the magnetization parallel to the easy axes. This reversal mechanism is called nucleation $[12,8]$ and the energy barrier is due to the domain wall energy. In the case of low anisotropy the particle rotates coherently. Here, all spins of the particle rotate in parallel and the energy barrier of the process is due to the anisotropy of the system $[13,14,8]$.

Results obtained for the lifetime of the metastable state and for the energy barriers which have to be overcome during the reversal have been reported earlier in detail $[8,10]$. In this paper we will mainly describe and discuss the special algorithm which we used in these investigations.

In our Monte Carlo procedure one single spin flip consists of three parts. At the beginning a spin is chosen randomly and a trial step of the spin is made (this trial step will be describe in detail below). Then the change of the energy of the system is computed according to Eq. (1). Finally the trial step is accepted with the usual probability from the heat-bath algorithm. Scanning the lattice and performing the procedure explained above once per spin (on average) is called one Monte Carlo step (MCS). It defines the time scale of the simulation. As we will show in the following the way the trial step is chosen is of importance for the efficiency of the algorithm as well as for the physical interpretation of the algorithm.

In our algorithm we use a combination of three different kinds of trial steps. The small trial step (STS) is the step which is usually used in models with continuous degrees of freedom [15]. It is a movement of the spin within a cone with a given opening angle around the initial spin direction. We adjusted this opening angle to roughly $30^{\circ}$. Thus each spin can only move by small steps and, hence, has to overcome the anisotropy energy barrier. This step can efficiently simulate the coherent rotation, but not the reversal by nucleation. If one is interested in the crossover from Heisenberg to Ising-like behavior with increasing anisotropy one has to allow also for large steps which can overcome the anisotropy energy barrier. Otherwise the dynamics of the system would freeze: in a system with very large anisotropy (Ising limit) no spin flip would occur at all.

In order to simulate also the nucleation, we use therefore another trial step, namely the uniform trial step (UTS). In this trial step an arbitrary spin direction is chosen at random which does not depend on the initial direction of the spin. This step samples the whole phase space efficiently. With this trial step a single spin is not forced to overcome the anisotropy energy barrier. Instead it is allowed to change from one (meta-) stable direction to the other immediately.

In order to simulate nucleation processes even more efficiently in the limit of very large anisotropy we also use a third trial step which we call reflection trial step (RTS) in the following. This step performs a reflection of the spin direction at the $x-y$-plane. It guarantees that the update algorithm crosses over to an efficient simulation of an Ising-like system for large anisotropy. Note, that this algorithm in general does not sample the whole phase space in contrast to the two algorithms mentioned before. Hence, it can only be used in combination with (at least) one of the other algorithms.

Therefore, we also consider an algorithm which performs a combined sampling: For each Monte Carlo step we use one of the different trial steps mentioned above and our update algorithm then consists of a series of Monte Carlo steps each using one of the three different trial steps explained above. We use three UTS, one STS and one RTS in such a series for all our simulations. This combination turned out to be most efficient.

In the following we show data of simulations for different values of the anisotropy constant using different algorithms namely the STS-algorithm and the UTS-algorithm and we compare it with simulations using the combined sampling. We simulate a cube of 


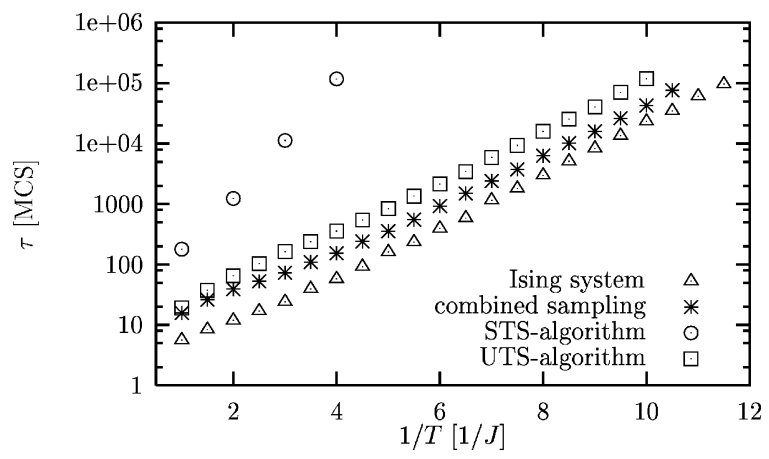

Fig. 1. Metastable lifetime $\tau$ vs. $1 / T$ for different algorithms $(d=2.5 \mathrm{~J} /$ spin, $B=0.5 \mathrm{~J})$ and comparison with the simulation of a corresponding Ising system.

8 spins with open boundary conditions. We start our simulations with an initial spin configuration where all spins are pointing up $\left(\mathbf{S}_{i}=(0,0,1)\right)$. The magnetic field $\mathbf{B}=(0,0,-B)$ destabilizes the system and after some time the magnetization will reverse. The metastable lifetime $\tau$ is defined by the condition $M_{z}(\tau)=0$ where $M_{z}$ is the $z$-component of the magnetization $\mathbf{M}=(1 / N) \sum_{i} \mathbf{S}_{i}$ (for details see $[8,10]$ ).

Fig. 1 shows the temperature dependence of the metastable lifetime for $d=2.5 \mathrm{~J} / \mathrm{spin}$ and $B=0.5 \mathrm{~J}$ comparing different algorithms. In the limit of large anisotropy the Heisenberg system should behave Isinglike, so it is straightforward to compare the simulation of the Heisenberg system with a direct simulation of a corresponding Ising system. The figure demonstrates that the UTS-algorithm and the combined sampling yield the same activation energy (the slope for low temperatures, see Eq. (2)) as the Ising system. The combined-sampling-algorithm has a lower lifetime than the UTS-algorithm since it contains the RTS which is most favorable in the Ising-limit. Hence, it is more efficient since it needs less computing time. With the STS-algorithm one does not obtain the activation energy for the nucleation process. Instead the slope is very large. It stems from an energy barrier which is caused by the anisotropy of each single spin. In the limit of infinite anisotropy the STS-algorithm would have to overcome an infinite energy barrier and, hence, no reversal would appear at all. We conclude that if one is interested in the crossover from a Heisenberg model to an Ising model with increasing anisotropy one cannot use an update algorithm which uses STS only.

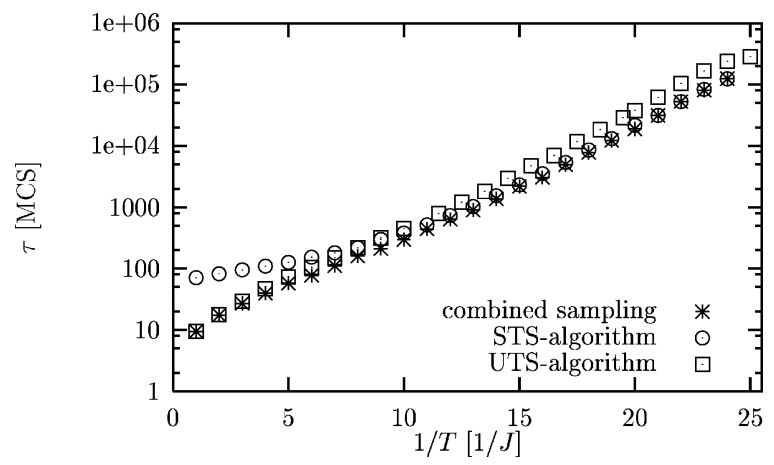

Fig. 2. Metastable lifetime $\tau$ vs. $1 / T$ for different algorithms $(d=0.5 \mathrm{~J} / \mathrm{spin}, B=0.5 \mathrm{~J})$.

Next we turn to the case of a lower anisotropy. Fig. 2 shows the temperature dependence of the metastable lifetime for an anisotropy $d=0.5 \mathrm{~J} / \mathrm{spin}$ and again $B=0.5 J$. As before we compare different algorithms. In this case the spins rotate coherently [8]. The figure demonstrates that for the STS-algorithm the values of the lifetime start at $70 \mathrm{MCS}$ for high temperature while in the case of the combined-sampling-algorithm and the UTS-algorithm these values are of the order of $10 \mathrm{MCS}$. Note that this offset is for every reversal mechanism the same (see Figs. 1 and 3). In the case of the STS-algorithm this offset is higher because due to the small steps the systems needs more MCS to reverse. For low temperature the data of the STSalgorithm and the combined sampling collapse. For all temperatures the combined-sampling-algorithm is efficient which makes it attractive for this kind of reversal mechanism also. Note also, that additionally one MCS of this algorithm needs less computing time than a MCS of the STS-algorithm because the UTS as well as the RTS need less arithmetic operations for each move of the spin.

Fig. 3 shows the temperature dependence of the metastable lifetime for anisotropy $d=0.3 \mathrm{~J} /$ spin and applied magnetic field $B=0.5 \mathrm{~J}$ as before. In this case the spins also rotate coherently, but the energy barrier is lower than before. Once again it is shown that in the limit of low temperature all algorithms yield the same activation barrier although it should be mentioned that for the UTS-algorithm the data are a little bit curved even in the limit of low temperatures. However, the algorithm with the smallest lifetimes is the STS-algorithm since the system overcomes the 


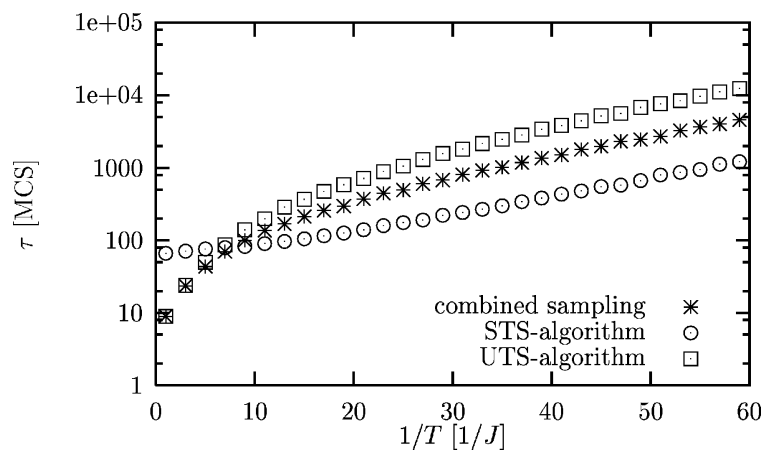

Fig. 3. Metastable lifetime $\tau$ vs. $1 / T$ for three different algorithms $(d=0.3 \mathrm{~J} / \mathrm{spin}, B=0.5 \mathrm{~J})$.

energy barrier with small steps. The combinationalgorithm in which energetically unfavorable trial steps are made (but not accepted) like the UTS or the RTS is obviously slower then the STS-algorithm.

To summarize, we compared different algorithms which use different kinds of trial steps for the Monte Carlo simulation of a classical Heisenberg system. We were especially interested in the crossover from magnetization reversal due to coherent rotation to nucleation with increasing uniaxial anisotropy. The STSalgorithm does not yield the energy barrier for a nucleation process but the energy barrier due to the anisotropy of each single spin. It is, hence, not appropriate for the study of the crossover to Ising-like behavior where the anisotropy becomes very large. In order to simulate all possible reversal processes efficiently we favor a combined-sampling-algorithm which performs different kinds of trial steps. The combined-sampling-algorithm is ergodic and it guarantees that all possible reversal mechanisms may occur in the system. Using this algorithm we can investigate the crossover from coherent rotation to nucleation. This algorithm yields the correct energy barrier and except for the case of very low anisotropy it is most efficient.

\section{Acknowledgments}

We thank K.D. Usadel for fruitful discussions. This work was supported by the Deutsche Forschungsgemeinschaft through Sonderforschungsbereich 166 and through the Graduiertenkolleg "Struktur und Dynamik heterogener Systeme".

\section{References}

[1] R.W. Chantrell, K. O’Grady, in: Applied Magnetism, R. Gerber, C.D. Wright, G. Asti (Eds.) (Kluwer Academic Publishers, Dordrecht, 1994) p. 113.

[2] C. Salling, R. O’Barr, S. Schultz, I. McFadyen, M. Ozaki. J. Appl. Phys. 75 (1994) 7986.

[3] M. Lederman, S. Schultz, M. Ozaki, Phys. Rev. Lett. 73 (1994) 1986.

[4] W. Wernsdorfer, K. Hasselbach, D. Mailly, B. Barbara, A. Benoit, L. Thomas, G. Suran, J. Mag. Mag. Mat. 140 (1995) 389 ;

W. Wernsdorfer, E. Bonet Orozco, K. Hasselbach, A. Benoit, B. Barbara, N. Demoncy, A. Loiseau, H. Pascard, D. Mailly, Phys. Rev. Lett. 78 (1997) 1791.

[5] W.T. Coffey, D.S.F. Crothers, J.L. Dormann, Yu.P. Kalmykov, E.C. Kennedey, W. Wernsdorfer, Phys. Rev. Lett. 80 (1998) 5655.

[6] P.A. Rikvold, B.M. Gorman, in: Annual Reviews of Computational Physics I, D. Staufer (Ed.) (World Scientific, Singapore, 1994) p. 149.

[7] H.L. Richards, M. Kolesik, P.A. Lindgård, P.A. Rikvold, M.A. Novotny, Phys. Rev. B 55 (1997) 11521.

[8] D. Hinzke, U. Nowak, Phys. Rev. B 58 (1998) 265.

[9] M. Acharyya, D. Stauffer, to be published in Eur. Phys. J. B.

[10] U. Nowak, D. Hinzke, submitted to J. Appl. Phys.

[11] J.M. Gonzáles, R. Ramírez, R. Smirnov-Rueda, J. González, Phys. Rev. B 52 (1995) 16034.

[12] R. Becker, W. Döring, Ann. Phys. (Leipzig) 24 (1935) 719.

[13] L. Néel, Ann. Geophys. 5 (1949) 99.

[14] W.F. Brown, Phys. Rev. 130 (1963) 1677.

[15] K. Binder, D.W. Heermann, Monte Carlo Simulation in Statistical Physics (Springer, Berlin, 1988) p. 21. 